\title{
Indigo plant leaf extract inhibits the binding of SARS-CoV-2 spike protein to angiotensin-converting enzyme 2
}

\author{
MAN HAGIYAMA $^{1 *}$, FUKA TAKEUCHI $^{1 *}$, AKI SUGANO $^{2}$, AZUSA YONESHIGE $^{1}$, TAKAO INOUE $^{1}$, \\ AKIHIRO WADA $^{1}$, HIROSHI KAJIYAMA ${ }^{1}$, YUTAKA TAKAOKA ${ }^{3,4}$, KENROH SASAKI $^{5}$ and AKIHIKO ITO ${ }^{1}$ \\ ${ }^{1}$ Department of Pathology, Kindai University Faculty of Medicine, Osaka-Sayama, Osaka 589-8511; \\ ${ }^{2}$ Center for Clinical Research, Toyama University Hospital; ${ }^{3}$ Data Science Center for Medicine and \\ Hospital Management, Toyama University Hospital, Toyama, Toyama 930-0194; ${ }^{4}$ Department of Medical Systems, \\ Kobe University Graduate School of Medicine, Kobe, Hyogo 650-0017; ${ }^{5}$ Division of Pharmacognosy, \\ Tohoku Medical and Pharmaceutical University, Sendai, Miyagi 981-8558, Japan
}

Received November 6, 2021; Accepted January 5, 2022

DOI: $10.3892 / \mathrm{etm} .2022 .11200$

\begin{abstract}
Severe acute respiratory syndrome coronavirus 2 (SARS-CoV-2) uses its $\mathrm{S} 1$ spike protein to bind to angiotensin-converting enzyme 2 (ACE2) on human cells in the first step of cell entry. Tryptanthrin, extracted from leaves of the indigo plant, Polygonum tinctorium, using $d$-limonene $(17.3 \mu \mathrm{g} / \mathrm{ml})$, is considered to inhibit ACE2-mediated cell entry of another type of coronavirus, HCoV-NL63. The current study examined whether this extract could inhibit the binding of the SARS-CoV-2 spike protein to ACE2. Binding was quantified as cell-bound fluorescence intensity in live cell cultures in which canine kidney MDCK cells overexpressing ACE2 were incubated with fluorescein-labeled $\mathrm{S} 1$ spike protein. When indigo extract, together with $\mathrm{S} 1$ protein, was added at $8,650 \mathrm{x}$ and 17,300x dilutions, fluorescence intensity decreased in a dose- and S1 extract-dependent manner, without affecting cell viability. When 4.0-nM tryptanthrin was added instead of the indigo extract, fluorescence intensity also decreased, but to a lesser degree than with indigo extract. Docking simulation analyses revealed that tryptanthrin readily bound to the receptor-binding domain of the $\mathrm{S} 1$ protein, and identified 2- and 7-amino acid sequences as the preferred binding sites. The indigo extract appeared to inhibit S1-ACE2 binding at high dilutions, and evidently contained other inhibitory
\end{abstract}

Correspondence to: Professor Akihiko Ito, Department of Pathology, Kindai University Faculty of Medicine, 377-2 Ohno-higashi, Osaka-Sayama, Osaka 589-8511, Japan

E-mail: aito@med.kindai.ac.jp

"Contributed equally

Key words: natural product, coronavirus disease 2019 prevention, severe acute respiratory syndrome coronavirus 2 spike protein, tryptanthrin, indigo plant, $d$-limonene, docking simulation elements as well as tryptanthrin. This extract may be useful for the prevention or treatment of SARS-CoV-2 infection.

\section{Introduction}

Severe acute respiratory syndrome coronavirus 2 (SARS-CoV-2) is responsible for coronavirus disease 2019 (COVID-19), and infects human cells by binding to human angiotensin-converting enzyme 2 (ACE2) with its spike protein $(1,2)$. The SARS-CoV-2 spike protein is composed of the $S 1$ receptor-binding subunit and the $S 2$ fusion subunit (2), and the spike resides on the viral surface as a trimer of this protein (2). The spike S1 subunit enables binding to ACE2 and its portion for the binding is called the receptor-binding domain (RBD) (3). Therefore, compounds with high affinity for RBD, if they can interfere with spike protein-ACE2 binding, are expected to be promising candidates for prophylactics against SARS-CoV-2 infection.

Leaves of the indigo plant, Polygonum tinctorium, have long been used for dyeing clothes in Japan. This leaf is not only an excellent source of blue dye, but has also proven to have anti-viral, anti-inflammatory, and anti-allergic activities (4-6). In parallel with these studies, remarkable progress has been made in identifying bioactive compounds in the leaves. One of the active components is tryptanthrin, indolo[2,1-b] quinazolin-6,12-dione. Interestingly, Tsai et al reported that tryptanthrin has antiviral action against human coronavirus NL63 (HCoV-NL63) (7). They incubated simian LLC-MK2 and human Calu-3 cells with HCoV-NL63, and found that the number of cells containing the virus was reduced by more than $80 \%$ in the presence of tryptanthrin, suggesting that tryptanthrin might kill the virus and/or interfere with viral entry into the cells. Importantly, HCoV-NL63 also binds to ACE2, although SARS-CoV-2 and HCoV-NL63 appear to have distinct binding sites on ACE2. We hypothesized that indigo plant leaf components might inhibit SARS-CoV-2 binding to ACE2.

In order to extract active components from indigo plant leaves, we devised an original extraction method using the 
solvent, $d$-limonene, (+)-p-Mentha-1,8-diene, an acyclic monoterpene widely used as a fragrance. The resulting indigo extract contains tryptanthrin at a fairly high concentration.

In the present study, with reference to a deposited preprint by Kapczynski et al (unpublished data), we prepared canine kidney epithelial MDCK cells overexpressing ACE2 and established a cell culture system that allowed us to quantify the degree of fluorescein-labeled S1 spike protein-ACE2 binding by measuring fluorescence intensity. We also conducted computer simulation analyses of docking between tryptanthrin and the spike protein.

\section{Materials and methods}

Cells and reagents. Madin-Darby canine kidney (MDCK) cells were purchased from the American Type Culture Collection (NBL-2) and cultured in Eagle's minimal essential medium with $10 \%$ fetal calf serum, as described in our previous report (8). Human colon adenocarcinoma Caco-2 cells were previously purchased from the Riken BioResource Center (RCB0988) (9). Tryptanthrin (SML0310; Sigma-Aldrich; Merck KGaA) was dissolved in DMSO at a concentration of $1 \mathrm{mg} / \mathrm{ml}$ and then diluted 10-fold in ethanol (final concentration of the stock solution, $100 \mu \mathrm{g} / \mathrm{ml}$ ).

Plasmid construction and transfection. Total RNA was extracted from Caco-2 cells using Trizol reagent (Invitrogen; Thermo Fisher Scientific, Inc.), and first strand cDNA was reverse-transcribed using total RNA as a template with Superscript IV (Invitrogen; Thermo Fisher Scientific, Inc.). Human ACE2 full-length cDNA (NM_001371415.1) was obtained by polymerase chain reaction (PCR) where Caco-2 cDNA was used as a template with the primer set: forward, 5'-gtggatgtgatcttggctca-3' and reverse, 5'-caaaatcacctcaag aggaaaaa-3'. The PCR product was inserted into the pTA2 TA-cloning vector (Toyobo). After amplification, the insert was excised at the NotI and HincII sites, and then inserted into the pCX4pur vector (10) at the NotI and HpaI sites (pCX4pur-hACE2). The absence of mutations was verified by sequencing.

MDCK cells $\left(8 \times 10^{4}\right)$ were grown in a 6-cm dish to $60-70 \%$ confluence and were transfected with either pCX4pur-hACE2 or the empty pCX4pur vector (5 $\mu \mathrm{g}$ each) using the Lipofectamine 3000 (Invitrogen; Thermo Fisher Scientific, Inc.) according to the manufacturer's instructions. Cells were selected by resistance to puromycin for two weeks.

Plant materials. Leaves of Polygonum tinctorium were collected in September 2020 in Aomori Prefecture, Japan. A voucher specimen was deposited in the herbarium of the medicinal herbal garden of Tohoku Medical and Pharmaceutical University and identified by KS.

Preparation of indigo plant leaf extract and high-performance liquid chromatography (HPLC). We used our original extract, named 'AOMORI-BLUE extract', which has been patented in Japan (Japanese Patent no. 6389492). Powdered, air-dried leaves $(100 \mathrm{~g})$ were extracted with $1,200 \mathrm{ml}$ of $d$-limonene (Wako Pure Chemical Industries, Ltd.) at room temperature for $48 \mathrm{~h}$. After filtration, a pale-yellow extract was obtained, and the extract was subjected to HPLC. To analyze the composition, the extract was dissolved in ethanol $(5.0 \mathrm{mg} / \mathrm{ml})$ and passed over a COSMOSIL 5PE-MS column (i.d. 4.6x250 mm; Nacalai Tesque) (mobile phase $40 \% \mathrm{CH}_{3} \mathrm{CN}$; flow rate, $1.0 \mathrm{ml} / \mathrm{min}$; detection $254 \mathrm{~nm}$; temperature $25^{\circ} \mathrm{C}$ ). Data were collected with a SIC Chromatocorder12 (System Instruments Co., Ltd.). A tryptanthrin standard was purchased from Sigma-Aldrich Japan. All other reagents were purchased from Fujifilm-Wako Pure Chemicals, Co. In the present study, indigo extract was diluted 10 -fold in ethanol (stock solution). $d$-limonene was also diluted 10 -fold in ethanol as a control stock solution. For reference, indigo leaves were extracted with ethanol $(99.5 \%)$ according to the same procedures.

S1 proteins and fluorescein labeling. Recombinant protein SARS-CoV-2 S1 subunit tagged with mouse IgG2a Fc portion (S1N-C5257) and normal mouse IgG (mIgG; 140-09511) were purchased from ACROBiosystems and Fujifilm-Wako Pure Chemicals, Co., respectively. A Fluorescein Labeling Kit- $\mathrm{NH}_{2}$ (Dojindo) was used to conjugate fluorescein to the S1 protein and mouse $\operatorname{IgG}$, according to the manufacturer's instructions.

Detection and quantification of fluorescein fluorescence in MDCK cell cultures. $3 \times 10^{3}$ of transfected or untransfected MDCK cells were suspended in $10 \mu \mathrm{l}$ of culture medium, and were transferred separately into the bottom of the micro-Insert 4-Well (gasket) on a $\mu$-Dish (35 mm, high, no. 80406; ibidi $\mathrm{GmbH})$. After $3 \mathrm{~h}$ of incubation, $150 \mu \mathrm{l}$ of medium were poured into the insert to fill all wells with identical medium. The next day, fluorescein-labeled S1 or $\mathrm{mIgG}$ was added to the culture medium at a concentration of $3 \mu \mathrm{g} / \mathrm{ml}$. At the same time, indigo extract, $d$-limonene, or tryptanthrin was added at the indicated concentrations, depending upon the experiment. After cell cultures were incubated $24 \mathrm{~h}$, the micro-insert gasket was gently removed, and cells were washed $3 \mathrm{x}$ with culture medium. Then, the $\mu$-Dish was filled with $1 \mathrm{ml}$ of culture medium, and was placed on the microscope stage of a $\mathrm{C} 2+$ confocal laser scanning system (Nikon, Tokyo, Japan). Fluorescein fluorescent images were captured with a 40x objective lens and analyzed on the Nikon $\mathrm{C}^{+}$computer system. Fluorescein intensity (arbitrary units per unit area) was measured at five randomly selected high-power fields for each well using Analysis Controls tools. Cell cultures in wells on $\mu$-Dishes were prepared and measured in triplicate for each experimental group, and the mean and standard deviation of fluorescein intensities were calculated using ROI Statistics. Experiments were independently repeated three time with similar results.

Immunofluorescence. After having detected the fluorescence from S1 proteins, we fixed the cells in $\mu$-Dishes with methanol for $10 \mathrm{~min}$ at $-20^{\circ} \mathrm{C}$, and blocked them with $2 \%$ bovine serum albumin for $30 \mathrm{~min}$ at room temperature. At this time, no fluorescein fluorescence was detectable on cells in any dishes. Then, cells were incubated with an antibody against ACE2 (E-11, sc-390851; Santa Cruz Biotechnology, Inc.) overnight at $4^{\circ} \mathrm{C}$, and were visualized with Alexa Flour 488-conjugated secondary antibody (anti-mouse IgG; Jackson ImmunoResearch). After washing with phosphate-buffered saline (PBS) three times, nuclei were labeled with DAPI 
(Molecular Probes) for $2 \mathrm{~h}$ at $4^{\circ} \mathrm{C}$. Fluorescent images were captured using a $\mathrm{C}^{+}$confocal scanning system equipped with 488-nm argon and 543-nm helium-neon lasers (Nikon).

Water-soluble tetrazolium- 8 assay. Cell viability was assessed with a water-soluble tetrazolium-8 (WST-8)-based colorimetric assay using a Cell Counting Kit 8 (Dojindo, Kumamoto, Japan). MDCK cells $\left(3 \times 10^{4}\right)$ were seeded in a 96-well plate in triplicate overnight, and then indigo extract was added to each well at indicated dilution rates. The next day, cells were incubated with WST-8 for $30 \mathrm{~min}$, and the absorbance was measured at $450 \mathrm{~nm}$ using an automated microplate reader. Measurement of mitochondrial dehydrogenase cleavage of WST- 8 to formazan dye provided an indication of cell viability.

Western blotting analysis. Cells were washed in PBS and were lysed in a buffer containing $50 \mathrm{mM}$ Tris- $\mathrm{HCl}(\mathrm{pH} 8.0)$, $150 \mathrm{mM} \mathrm{NaCl}, 1 \%$ Triton X-100 and $1 \mathrm{mM}$ phenylmethylsulfonyl fluoride. After removal of impurities by centrifugation, lysates were subjected to Western blot analyses, as described in our previous report (11). Immunoreactive band intensities were quantified using ImageJ software (National Institutes of Health), as described previously (12).

Molecular simulation. We obtained the 3D structure of the of SARS-CoV-2 spike protein trimer from the Protein Data Bank (https://www.rcsb.org/) (PDB ID: 6Z97). We selected this structure because it satisfies the following conditions: i) the receptor-accessible 'up' conformation is available in the trimer, ii) the amino acid sequence is conserved without deletions, and, iii) the structure is available at a resolution $<3.5 \AA$.

The 3D structure of tryptanthrin was downloaded from the PubChem database (https://pubchem.ncbi.nlm.nih.gov) (CID: 73549; no other conformers are known). Using these structures, we simulated 100 docking runs for the spike protein with tryptanthrin using AutoDock 4.2 in the grid box surrounding the Receptor Binding Motif (RBM). We then analyzed the number of dockings to the ACE2 binding site in the binding mode.

Statistical analysis. Comparative analyses were done on experiments consisting of more than two groups. We compared fluorescence intensities using one-way analysis of variance (ANOVA). We calculated the mean of each group, and compared mean values between two groups using the Bonferroni correction of one-way ANOVA. WST-8 data were analyzed with Dunnett's multiple comparison test. Comparisons between two groups were done with Student's t-test. P-values $\leq 0.05$ were considered statistically significant. Correlations between cell densities and CADM1 protein levels using Spearman's rank test were considered significant if $R^{2} \geq 0.1$ and $\mathrm{P} \leq 0.05$.

\section{Results}

HPLC of indigo extract. An HPLC peak with a retention time of 26.328 min was identified as tryptanthrin, the concentration of which was estimated at $17.3 \mu \mathrm{g} / \mathrm{ml}$ (Fig. 1). This concentration

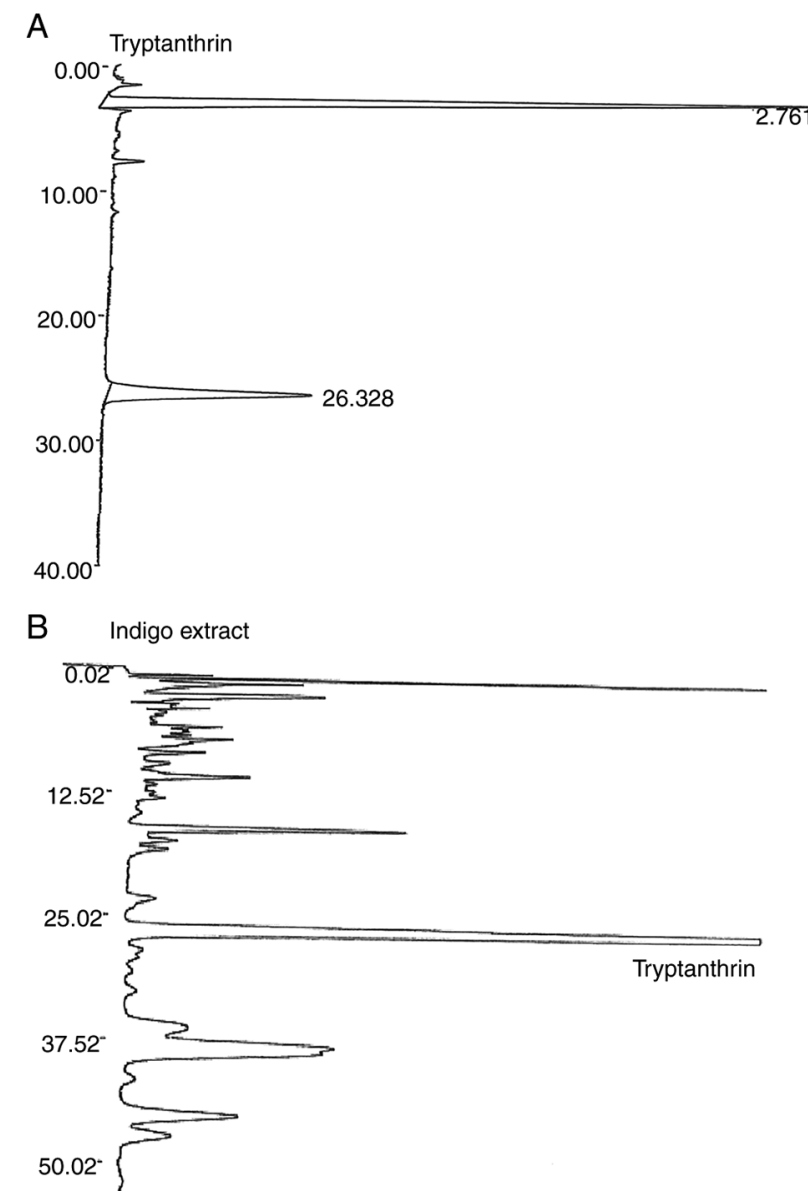

Figure 1. high performance liquid chromatography analysis of indigo extract. (A) Tryptanthrin standard $(10.0 \mu \mathrm{g} / \mathrm{ml})$. (B) Indigo extract (tryptanthrin content, $17.3 \mu \mathrm{g} / \mathrm{ml}$ ).

was much higher than that in the extract prepared using ethanol (compare Figs. 1 and S1). There were other high peaks at retention times from 13-20, 35-40 and 45-50 min. These peaks could not be recognized as known compounds.

Detection of S1 protein bound to ACE2 on MDCK cells. We transfected MDCK cells with the pCX4pur vector carrying the human ACE2 full-length cDNA and the empty vector, and we obtained cells that robustly expressed ACE2 (MDCK-ACE2) and the vector-control cells (MDCK-vector), respectively. Expression of ACE2 was confirmed by immunofluorescence and Western blotting analyses. An anti-ACE2 antibody clearly detected ACE2 expression on cell membranes of MDCK-ACE2 cells, and detected it as a $\sim 150-\mathrm{kDa}$ band in the cell lysate from MDCK-ACE2 cells, but not from MDCK-vectors or untransfected MDCK cells (Fig. 2A and B).

We tried to quantitatively detect binding of S1 proteins to ACE2 in live cell cultures. For this purpose, we used S1 proteins fused with mouse $\mathrm{IgG} \mathrm{Fc}$, and labeled them with fluorescein. The resulting protein (S1-Fc-fluorescein) was added to untransfected MDCK, MDCK-vector, and MDCK-ACE2 cell culture in $\mu$-Dishes at a concentration of $3 \mu \mathrm{g} / \mathrm{ml}$. After $24 \mathrm{~h}$, live cells were washed with PBS and observed through a confocal laser microscope. Fluorescein fluorescence was clearly detected on cell membranes of MDCK-ACE2 cells, but was not seen in untransfected MDCK or MDCK-vector 
A

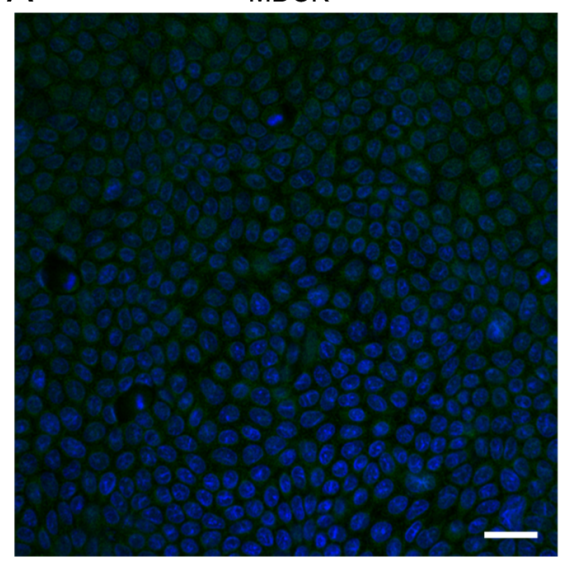

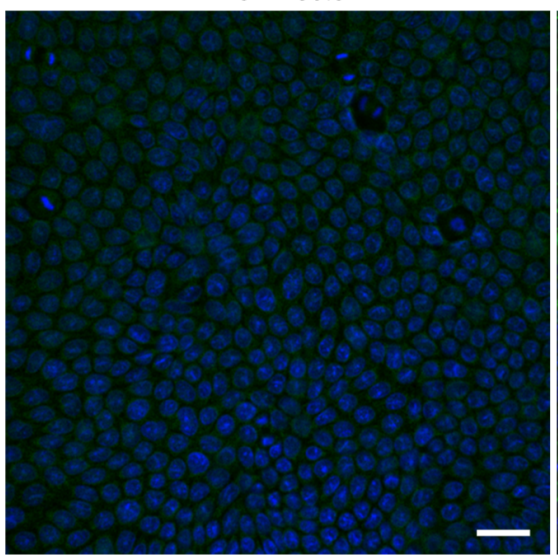

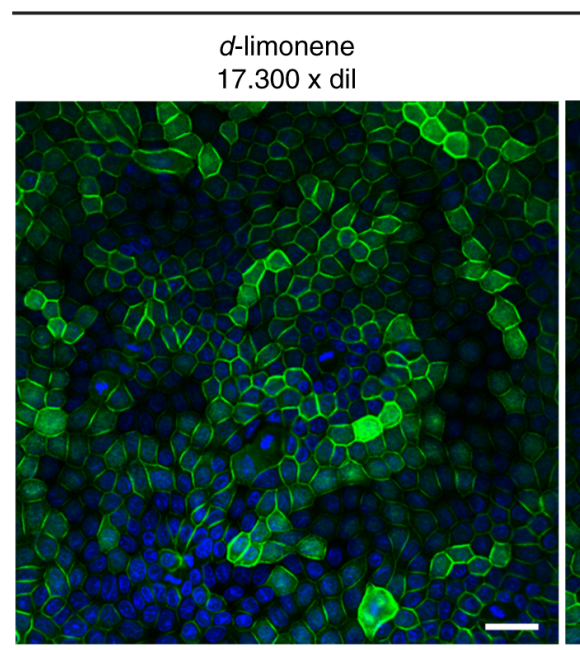

B

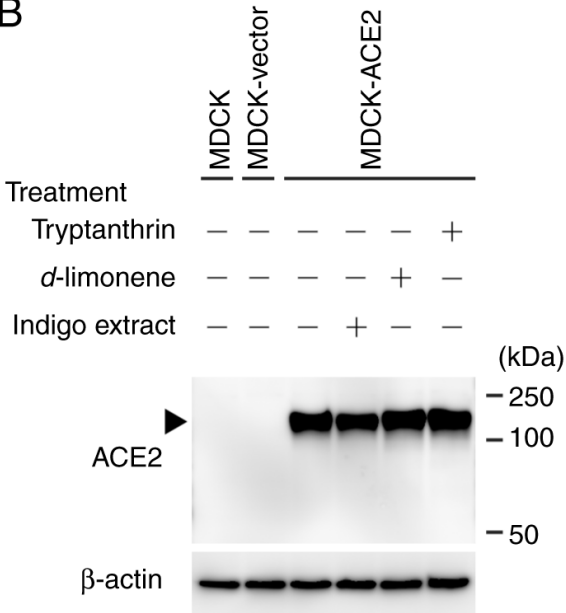

MDCK-vector

MDCK-ACE2

MDCK-ACE2

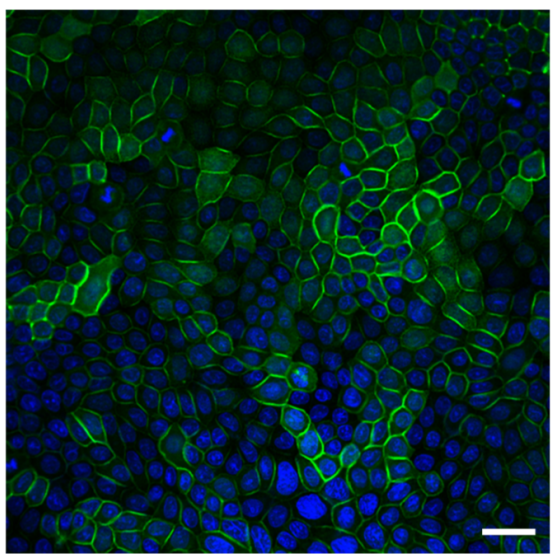

Tryptanthrin $1 \mathrm{ng} / \mathrm{ml}$

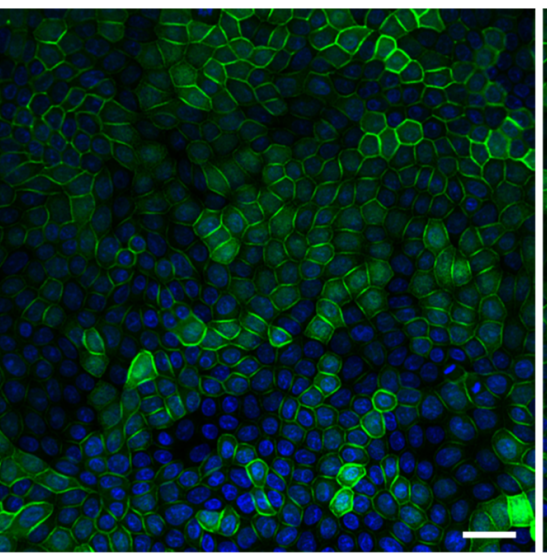

C

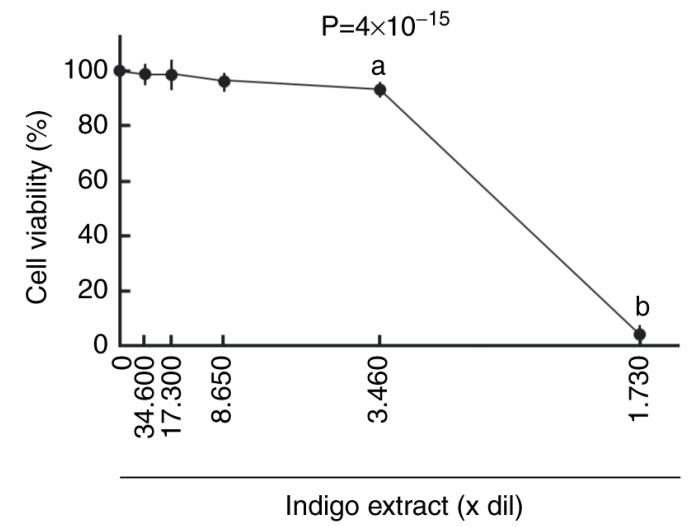

Figure 2. Expression of ACE2 in MDCK cells. (A) Immunofluorescence of untransfected MDCK, MDCK-vector and MDCK-ACE2 cells using an anti-ACE2 antibody (green; upper). In the lower panels, MDCK-ACE2 cells were incubated with indigo extract, tryptanthrin or $d$-limonene at indicated dilutions or concentrations, together with S1-Fc-fluorescein. Cell nuclei were labeled with DAPI (blue). Merged images of green and blue fluorescent signals are presented (scale bars $=50 \mu \mathrm{m}$ ). (B) Western blot analyses of various types of MDCK cells using an anti-ACE2 antibody. An arrowhead indicates ACE2-specific bands. The blot was re-probed with an anti- $\beta$-actin antibody to determine the amount of protein loading per lane. (C) WST- 8 assays of MDCK cellsin the presence of serially diluted indigo extract. Viability percentages are line-plotted by dots with bars indicating the mean and standard deviation from triplicate wells, respectively. P-values from one-way ANOVA are shown above the graph. ${ }^{\mathrm{a}} \mathrm{P}=0.0168$ and ${ }^{\mathrm{b}} \mathrm{P}<0.001$ vs. $\mathrm{X}=0$ (absence of extract). ACE2, angiotensin-converting enzyme 2; dil, dilution; MDCK, Madin-Darby canine kidney.

cells (Figs. 3A and S2). For a negative control, mouse IgG was labeled with fluorescein, and added to the cultures. No fluorescence was detected in either type of cells (Fig. S3). Fluorescent intensities were calculated for each cell type and treatment setting. MDCK-ACE2 cells incubated with S1-Fc-fluorescein had much higher fluorescence intensity than the other types of cultures $\left(\mathrm{P}<2 \times 10^{-16}\right.$; Figs. $3 \mathrm{~A}$ and $\mathrm{S} 3$ and Table SI). 
A

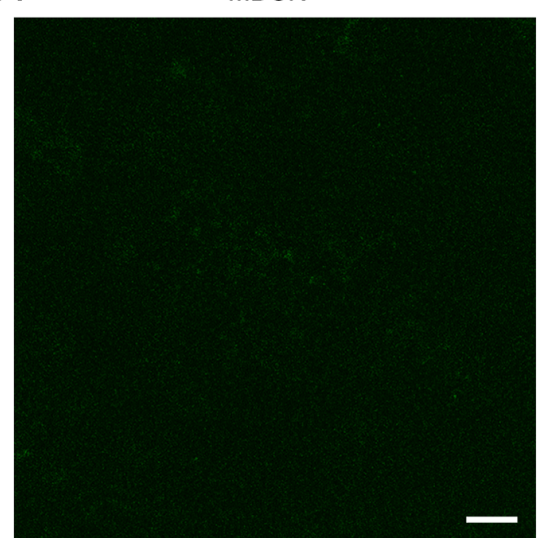

$636 \pm 42.52$

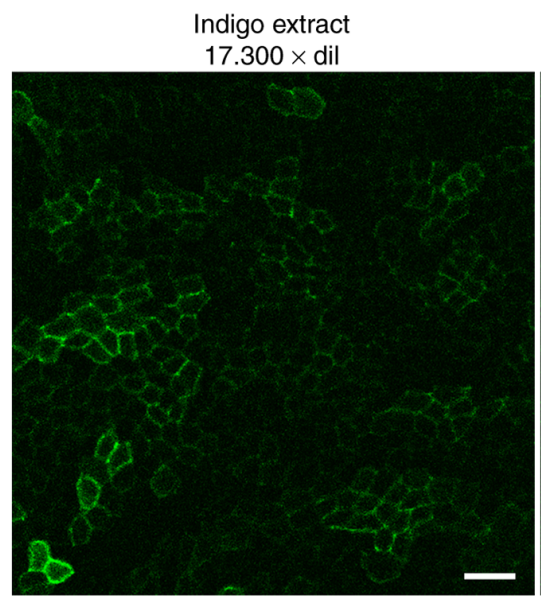

$1103 \pm 76.83^{a, d}$
MDCK-vector

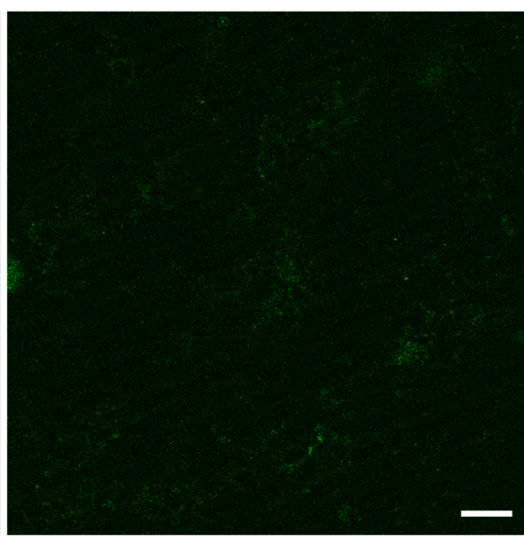

$706 \pm 53.50$

Tryptanthrin

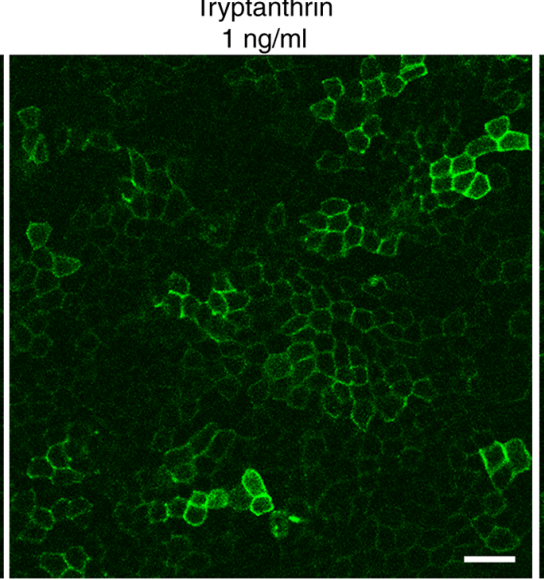

$1359 \pm 104.35^{b}$
MDCK-ACE2

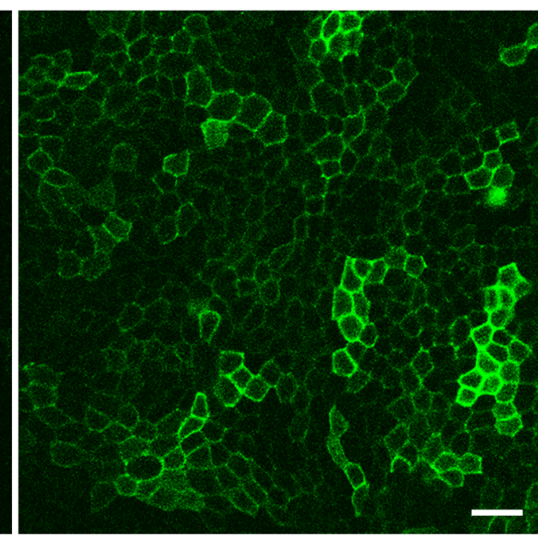

$1611 \pm 184.48$

Indigo extract $86.500 \times$ dil

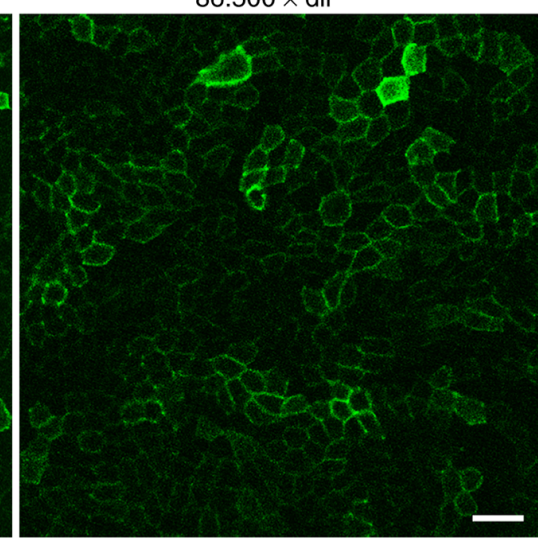

$1510 \pm 81.18^{c}$

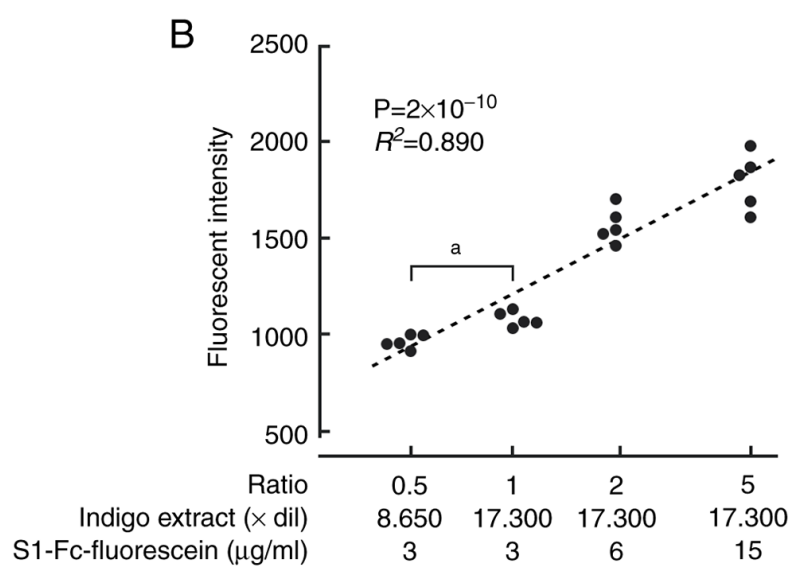

Figure 3. Quantification of S1 proteins bound to ACE2 on MDCK cells. (A) MDCK, MDCK-ACE2 and MDCK-vector cells were incubated with S1-Fc-fluorescein $(3 \mu \mathrm{g} / \mathrm{ml})$. In some MDCK-ACE2 cell cultures, either indigo extract or tryptanthrin was also added at indicated dilution rates or concentrations. After one day of incubation and wash, fluorescent intensity of fluorescein remaining on the cells was measured using a confocal laser microscopy system. Representative photomicrographs are presented. Below each image, the mean and standard deviation of the intensity (arbitrary unit) are presented for the corresponding experimental group. ${ }^{\mathrm{a}} \mathrm{P}=1.20 \times 10^{-13},{ }^{\mathrm{b}} \mathrm{P}=0.0033$ and ${ }^{\mathrm{c}} \mathrm{P}>0.999$ vs. MDCK-ACE2 cell intensity; ${ }^{\mathrm{d}} \mathrm{P}=0.0027$ vs. tryptanthrin treatment (scale bars $=50 \mu \mathrm{m})$. (B) In MDCK-ACE2 cell cultures, various ratios of concentrations of indigo extract and S1-Fc-fluorescein were used. The ratio was expressed as 1 under the conditions in A (indigo extract, 17,300-fold dilution; S1-Fc-fluorescein, $3 \mu \mathrm{g} / \mathrm{ml}$ ). The ratio (logarithmic in X-axis) and fluorescent intensity (linear in Y-axis) are presented in a scatter plot ( $\mathrm{n}=5$ for each ratio group). The dot distribution approximates a linear function (dotted lines). Correlations and statistical significance were analyzed using Spearman's rank test. $R^{2}$ and P-values are presented. ${ }^{a} \mathrm{P}=3 \times 10^{-4}$ as indicated. ACE2, angiotensin-converting enzyme 2; MDCK, Madin-Darby canine kidney.

Inhibition of S1-ACE2 binding by indigo extract. WST-8 assays revealed that indigo extract had no substantial effect on MDCK cell viability when diluted $\geq 8,650$-fold (Fig. $2 \mathrm{C}$ ). The extract did not change the medium $\mathrm{pH}$ in this range of dilution
(4.39-7.44). Together with S1-Fc-fluorescein, the indigo extract stock solution was added to confluent MDCK-ACE2 cell cultures in $\mu$-Dishes at a dilution of 1,730-fold (17,300-fold for the original extract), and after $24 \mathrm{~h}$, cells were observed 
Table I. Docking results of tryptanthrin-S1 spike protein docking run per 100 times.

\begin{tabular}{lccc}
\hline \multirow{2}{*}{$\begin{array}{l}\text { No. of correct } \\
\text { binding models }\end{array}$} & \multicolumn{3}{c}{ Docking score } \\
\cline { 2 - 4 } & Mean \pm SD & Most stable & Score ranking \\
\hline 58 & $-5.88 \pm 0.10$ & -6.02 & 26 \\
\hline
\end{tabular}

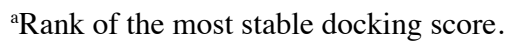

Table II. Docking results of the same binding mode in 58 correct tryptanthrin-S1 spike protein bindings.

\begin{tabular}{lcc}
\hline $\begin{array}{l}\text { No. of the same } \\
\text { binding mode }\end{array}$ & $\begin{array}{c}\text { Amino acids } \\
\text { targeted }\end{array}$ & $\begin{array}{c}\text { Docking score } \\
(\text { mean } \pm \text { SD) }\end{array}$ \\
\hline 45 & $455-456$ and $488-494$ & $-5.88 \pm 0.02$ \\
\hline
\end{tabular}

alive. Fluorescent signals were weakly detectable (Fig. 3A). The fluorescence intensity was much lower than that in MDCK-ACE2 cell cultures without indigo extract $\left(\mathrm{P}<2 \times 10^{-16}\right.$; Fig. 3A and Table SI).

Since indigo extract contained tryptanthrin at a concentration of $17.3 \mu \mathrm{g} / \mathrm{ml}$ (Fig. 1), the tryptanthrin concentration was estimated to be $1.0 \mathrm{ng} / \mathrm{ml}$ in the above treatment. Instead of indigo extract, we added tryptanthrin (molecular weight 248.24) alone, together with S1-Fc-fluorescein, to MDCK-ACE2 cell cultures at a concentration of $1.0 \mathrm{ng} / \mathrm{ml}$, or $4.0 \mathrm{nM}$. The fluorescent intensity of fluorescein decreased, but the degree of diminution was smaller than that caused by indigo extract (Fig. 3A).

Next, we used indigo extract with a further 5 -fold dilution, i.e., an 86,500-fold dilution. The inhibitory effect on S1-ACE2 binding substantially disappeared, suggesting that the effect of the indigo extract is dose-dependent (Fig. 3B). Then, we changed the ratio of concentrations of indigo extract and S1-Fc-fluorescein added to the culture. There was a significant correlation between the ratio and S1 fluorescent intensity. That is, higher relative concentrations of indigo extract resulted in decreased S1 fluorescence intensity, while higher ratios of S1 increased S1 fluorescent intensity (Fig. 3B).

We conducted Western blotting analyses and immunofluorescence on MDCK-ACE2 cells treated with indigo extract or $d$-limonene at a 17,300 -fold dilution and tryptanthrin at $1.0 \mathrm{ng} / \mathrm{ml}$. Both experiments revealed that ACE2 expression was essentially unchanged with either treatment (Fig. 2A).

Docking simulation of S1-ACE2 binding. Molecular simulation methods were used to examine possible mechanisms by which tryptanthrin inhibits binding of S1 protein to ACE2 on MDCK cells. We searched the PDB, drew the 3D structure of the spike protein trimer bound to ACE2 using PDB ID 6M0J, and obtained the 3D structure of the spike protein trimer PDB ID 6Z97 (Tables I and II and Fig. 4). We conducted docking simulation analyses to examine how tryptanthrin binds to RBM. We found that tryptanthrin molecules preferentially
Table III. Amino acid residues of SARS-CoV-2 S1 spike protein that are involved in binding to ACE2 or tryptanthrin.

\begin{tabular}{|c|c|}
\hline ACE2 amino acids & amino acids \\
\hline K417, G446, Y449, Y453, L455a, & $\mathrm{L} 455^{\mathrm{a}}, \mathrm{F} 456^{\mathrm{a}} \mathrm{E} 484$ \\
\hline $\mathrm{F} 456^{\mathrm{a}}$, A475, F486, N487, Y489 ${ }^{\mathrm{a}}$, & G485, C488, Y489a \\
\hline Q493', G496, Q498, T500, N501, & F490, L492 and \\
\hline G502 and Y505 & $\mathrm{Q}_{493^{\mathrm{a}}}$ \\
\hline
\end{tabular}

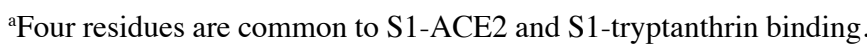
For tryptanthrin-S1 docking, the same binding mode was repeated 45 times in 58 correct binding runs (presented in Table I). Binding was defined by proximity of $4 \AA$ between S1 and ACE2 or S1 and tryptanthrin. ACE2, angiotensin-converting enzyme 2 .

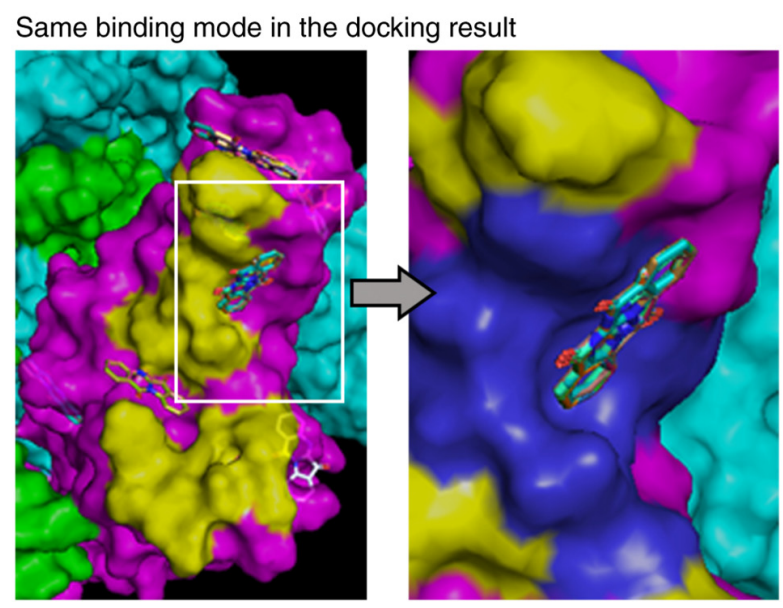

Figure 4. Same binding mode of tryptanthrin-S1 docking. Magenta, receptor binding domain; yellow, reported residues contacting to ACE2; blue, amino acids targeted in the same binding mode

bound to the ACE2 binding site reported in 6M0J (13) in more than half of the 100 docking runs (Tables I and II). Notably, most of this binding showed the same binding mode, in which the molecular plane of tryptanthrin was nearly perpendicular to the surface of RBM around Leu455-Phe456 and Cys488-Ser494 (Fig. 4 and Table III). In these two regions, S1 spike protein was simulated to have four amino acids that are involved in binding to ACE2 and tryptanthrin (Table III).

\section{Discussion}

In the present study, we established a cell culture assay system which enabled us to easily quantify the amount of SARS-CoV-2 spike protein bound to ACE2 on mammalian cells. This system appeared useful to assess the inhibitory effect on this binding by various reagents. We found that this binding was inhibited by indigo extract prepared using $d$-limonene as a solvent (molecular weight 136.23; specific gravity $0.842 \mathrm{~g} / \mathrm{ml}$ ). $d$-limonene reportedly suppresses mammalian cell viability and growth, depending on its concentration. Past studies demonstrate that $d$-limonene has no effect on PC12 cell viability and DU-145 and PZ-HPV-7 cell survival 
even at $0.6 \mu \mathrm{l} / \mathrm{ml}(1,667$-fold $\mathrm{v} / \mathrm{v}$ dilution) and $0.5 \mathrm{mM}$, respectively $(14,15)$. We used indigo extract mainly at a 17,300-fold v/v dilution, i.e., $0.35 \mathrm{mM} d$-limonene. The WST-8 assay showed that this concentration was low enough for MDCK cells to remain healthy; therefore, $d$-limonene was useful for indigo leaf extraction. Actually, we did not detect substantial changes in ACE2 expression in MDCK cells treated with indigo extract or $d$-limonene (Fig. 2A). This also supported the notion that indigo extract inhibited S1-ACE2 binding, not ACE2 expression, at high dilution rates.

The present study also showed that tryptanthrin inhibited S1-ACE2 binding, though it did not account for the entire inhibitory effect of indigo extract. Tryptanthrin was reported to reduce the HCoV-NL63 infectivity with $\mathrm{IC}_{50}$ values of 0.30 and $1.52 \mu \mathrm{M}$ in Calu-3 and LCC-MK2 cells, respectively (7). We used tryptanthrin at concentrations of $4.0 \mathrm{nM}$, suggesting that tryptanthrin may be much more effective against SARS-CoV-2. This speculation, however, may be too simple, because we examined the inhibitory effect only on S1-ACE2 binding, not infectivity, in MDCK cells that had been forced to overexpress ACE2. To the best of our knowledge, the minimal $50 \%$ cytotoxic concentration $\left(\mathrm{CC}_{50}\right)$ of tryptanthrin is $173.2 \mu \mathrm{M}$ for Calu-3 cells (7), and tryptanthrin is generally thought to have no significant cytotoxicity to human normal cells $(16,17)$. In terms of cytotoxicity, tryptanthrin can be expected to serve as an inhibitor for S1-ACE2 binding within its safe concentration range.

Since tryptanthrin is much smaller than $\mathrm{S} 1$ protein, one may wonder how it can inhibit binding. Docking simulation analyses revealed that tryptanthrin bound to the RBM of the spike protein trimer mainly using nine amino acid residues, four of which are involved in S1 RBM-ACE2 binding, and its molecular plane was nearly perpendicular to the RBM surface. This binding conformation may explain why tryptanthrin inhibits the binding of S1 protein to ACE2. Even though it is small, tryptanthrin may bind to residues essential for RBM-ACE2 binding. Consistent with this simulation, the competitive nature of indigo extract was illustrated by cell culture experiments in which we changed the ratios of concentrations of the extract and $\mathrm{S} 1$ protein added to the cultures (Fig. 3B). However, another possibility remains. Tryptanthrin may have a stronger affinity for ACE2 than S1 protein. Since ACE2 is crucial to heart function control (18), this possibility must be examined carefully when clinical applications are considered.

In addition, the present study suggests that indigo extract contains other active components beside tryptanthrin. Compared with ethanol, $d$-limonene extracts low-polarity components, including tryptanthrin, rather than high-polarity components such as glycosides (compare Figs. 1 and S2). With this feature in mind, we are now trying to isolate and identify the active components. Since Polygonum tinctorium is generally classified as food and its toxicity has not been reported, we are also developing in vivo experiments to administer indigo extract intranasally to mice. Pharmacokinetic analyses are planned on the extract and other active components.

In conclusion, we demonstrated that indigo extract has an inhibitory effect on binding of S1 to ACE2 at concentrations low enough not to affect cell viability. One of the active components appears to be tryptanthrin, but the extract likely contains other active, unidentified elements. Further investigation may open a new avenue for practical use of this natural product as a prophylactic against SARS-CoV-2 infection.

\section{Acknowledgements}

Not applicable.

\section{Funding}

This study was supported by the Japan Society for the Promotion of Science KAKENHI (grant nos. 17K08680, 20K07434, 18K07049 and 21K06978), the Takeda Science Foundation (to MH, 2019) and the All-Kindai University support project against COVID-19 (to AI, 2020 and 2021). The current study also received funding from Aomori AI Industrial Co., Ltd., Aomori, Japan.

\section{Availability of data and materials}

The datasets used and/or analyzed during the current study are available from the corresponding author on request.

\section{Authors' contributions}

$\mathrm{MH}$ and FT constructed expression vectors and performed transfection. $\mathrm{MH}$ and FT also conducted cell culture experiments, confocal microscopic studies and western blot analyses. AY, TI, HK and AW helped complete these experiments. KS provided plant materials and performed HPLC. AS and YT conducted simulation analyses. $\mathrm{MH}$ conducted the statistical analyses. MH, FT and AI confirmed the authenticity of all the raw data. KS and AI conceived and designed the study, and AI drafted the manuscript. All authors read and approved the final manuscript.

\section{Ethics approval and consent to participate}

Not applicable.

\section{Patient consent for publication}

Not applicable.

\section{Competing interests}

The authors declare no competing interests.

\section{References}

1. Huang C, Wang Y, Li X, Ren L, Zhao J, Hu Y, Zhang L, Fan G, $\mathrm{Xu}$ J, Gu X, et al: Clinical features of patients infected with 2019 novel coronavirus in Wuhan, China. Lancet 395: 497-506, 2020.

2. Walls AC, Park YJ, Tortorici MA, Wall A, McGuire AT and Veesler D: Structure, function, and antigenicity of the SARS-CoV-2 spike glycoprotein. Cell 181: 281-292.e6, 2020.

3. Wrapp D, Wang N, Corbett KS, Goldsmith JA, Hsieh CL, Abiona O, Graham BS and McLellan JS: Cryo-EM structure of the 2019-nCoV spike in the prefusion conformation. Science 367: 1260-1263, 2020 .

4. Zhong Y, Yoshinaka Y, Takeda T, Shimizu N, Yoshizaki S, Inagaki Y, Matsuda S, Honda G, Fujii N and Yamamoto N: Highly potent anti-HIV-1 activity isolated from fermented Polygonum tinctorium Aiton. Antiviral Res 66: 119-128, 2005. 
5. Ishihara T, Okura T, Kohno K, Tanimoto T, Ikegami $\mathrm{H}$ and Kurimoto M: Polygonum tinctorium extract suppresses nitric oxide production by activated macrophages through inhibiting inducible nitric oxide synthase expression. J Ethnopharmacol 72 : 141-150, 2000.

6. Han NR, Kang SW, Moon PD, Jang JB, Kim HM and Jeong HJ: Genuine traditional Korean medicine, Naju Jjok (Chung-Dae, Polygonum tinctorium) improves 2,4-dinitrofluorobenzene-induced atopic dermatitis-like lesional skin. Phytomedicine 21: 453-460, 2014.

7. Tsai YC, Lee CL, Yen HR, Chang YS, Lin YP, Huang SH and Lin CW: Antiviral action of tryptanthrin isolated from Strobilanthes cusia leaf against human coronavirus NL63. Biomolecules 10: 366, 2020.

8. Hosokawa $\mathrm{Y}$, Hagiyama M, Iino T, Murakami $\mathrm{Y}$ and Ito $\mathrm{A}$ Noncontact estimation of intercellular breaking force using a femtosecond laser impulse quantified by atomic force microscopy. Proc Natl Acad Sci USA 108: 1777-1782, 2011.

9. Hagiyama M, Yabuta N, Okuzaki D, Inoue T, Takashima Y, Kimura R, Ri A and Ito A: Modest static pressure suppresses columnar epithelial cell growth in association with cell shape and cytoskeletal modifications. Front Physiol 8: 997, 2017.

10. Kimura R, Otani T, Shiraishi N, Hagiyama M, Yoneshige A Wada A, Kajiyama H, Takeuchi F, Mizuguchi N, Morishita K and Ito A: Expression of cell adhesion molecule 1 in human and murine endometrial glandular cells and its increase during the proliferative phase by estrogen and cell density. Life Sci 283: 119854, 2021.

11. Koma Y, Furuno T, Hagiyama M, Hamaguchi K, Nakanishi M, Masuda M, Hirota S, Yokozaki $\mathrm{H}$ and Ito A: Cell adhesion molecule 1 is a novel pancreatic-islet cell adhesion molecule that mediates nerve-islet cell interactions. Gastroenterology 134: 1544-1554, 2008.
12. Mimae T, Okada M, Hagiyama M, Miyata Y, Tsutani Y, Inoue T, Murakami Y and Ito A: Upregulation of notch2 and six1 is associated with progression of early-stage lung adenocarcinoma and a more aggressive phenotype at advanced stages. Clin Cancer Res 18: 945-955, 2012.

13. Lan J, Ge J, Yu J, Shan S, Zhou H, Fan S, Zhang Q, Shi X, Wang Q, Zhang L and Wang X: Structure of the SARS-CoV-2 spike receptor-binding domain bound to the ACE2 receptor. Nature 581: 215-220, 2020.

14. Tang XP, Guo XH, Geng D and Weng LJ: d-Limonene protects PC12 cells against corticosterone-induced neurotoxicity by activating the AMPK pathway. Environ Toxicol Pharmacol 70: 103192, 2019.

15. Rabi T and Bishayee A: d-Limonene sensitizes docetaxel-induced cytotoxicity in human prostate cancer cells: Generation of reactive oxygen species and induction of apoptosis. J Carcinog 8: 9 , 2009.

16. Shankar GM, Alex VV, Nisthul AA, Bava SV, Sundaram S, Retnakumari AP, Chittalakkottu S and Anto RJ: Pre-clinical evidences for the efficacy of tryptanthrin as a potent suppressor of skin cancer. Cell Prolif 53: e12710, 2020.

17. Han NR, Kim HM and Jeong HJ: Tryptanthrin reduces mast cell proliferation promoted by TSLP through modulation of MDM2 and p53. Biomed Pharmacother 79: 71-77, 2016.

18. Crackower MA, Sarao R, Oudit GY, Yagil C, Kozieradzki I, Scanga SE, Oliveira-dos-Santos AJ, da Costa J, Zhang L, Pei Y, et al: Angiotensin-converting enzyme 2 is an essential regulator of heart function. Nature 417: 822-828, 2002.

This work is licensed under a Creative Commons Attribution-NonCommercial-NoDerivatives 4.0 International (CC BY-NC-ND 4.0) License. 